\title{
Minimization of Acoustic Potential Energy in Enclosure Using Both Active Noise Control and Active Vibration Control*
}

\author{
Kozue KOBAYASHI** and Nobuo TANAKA**
}

\begin{abstract}
This paper concerns the minimization of acoustic potential energy in an enclosure by both active noise control (ANC) and active vibration control (AVC). First, the optimal feedforward control law for minimizing the acoustic potential energy in a cavity walled by flexible structures is derived. Secondly, it is found that the control acoustic power radiated from each acoustic secondary source used for ANC and control vibration power infused by each control actuator for AVC become zero under the optimal control condition. It is also found that the zero control power phenomena are the necessary conditions for the optimality. Finally, the control effects for minimizing the potential energy using ANC and AVC are demonstrated from a viewpoint of numerical analysis.
\end{abstract}

Key Words: Active Noise Control, Active Vibration Control, Acoustic Potential Energy, Optimal Feed-Forward Control Law, Zero Control Power

\section{Introduction}

In recent mechanical designing, reducing noise from the structures is one of the most important problems to solve. The biggest cause of the noise may be vibration of the structures. There are two active control methods known as effective ways to reduce the noise; one is Active Noise Control $(\mathrm{ANC})^{(1),(2)}$ and the other is Active Vibration Control $(\mathrm{AVC})^{(3),(4)}$. In problems of noise in a cavity, Nelson et al. ${ }^{(5)-(7)}$ reported on reducing the noise radiated from acoustic point sources by ANC with acoustic point sources. Kim et al. ${ }^{(8)}$ and Snyder et al. ${ }^{(9),(10)}$ reported of the control effect for reducing the noise transmitted from the exterior into a cavity by ANC or AVC. However, no one reported the control effects provided by ANC and AVC in cases where the target enclosure consists of flexible and rigid walls and where the flexible walls vibrate and radiate the noise into the enclosure. Therefore, this paper presents the problem of minimization of acoustic potential energy in a cavity contained within flexible walls vibrated by some disturbance forces, and shows the effects achieved through ANC with some acoustic secondary sources placed in the cavity and AVC with some control actuators on the vibrating walls.

\footnotetext{
* Received 12th July, 2004 (No. 02-0796)

** Department of Mechanical Engineering, Tokyo Metropolitan Institute of Technology, 6-6 Asahigaoka, Hino-shi, Tokyo 191-0062, Japan.

E-mail: qd023803@cc.tmit.ac.jp
}

First, the optimal feedforward control law to minimize the acoustic potential energy is derived. Secondly, two phenomena which come to appear under the optimal control condition are illustrated; one is acoustic power radiated from each acoustic secondary source to become zero, and the other is vibration power infused into the walls by each control actuator to become zero. In addition, it is shown that the zero power phenomena are not the sufficient but necessary conditions for the optimal control condition. Moreover, from a viewpoint of a numerical simulation, the reduction of the acoustic potential energy in the cavity achieved by both ANC and AVC are demonstrated. Finally, the zero control power phenomena appearing under the optimal control condition are expounded on using intensity distributions.

\section{Optimal Control Law}

This chapter presents a way to derive the optimal control law of ANC and AVC to minimize acoustic potential energy in a cavity. The cavity of interest, depicted in Fig. 1, is surrounded with flexible walls excited by $N_{d}$ disturbance forces. The internal noise is produced by vibration of the walls. Controllers for ANC are $N_{s}$ acoustic point sources within the cavity, and for AVC are $N_{c}$ actuators on the wall.

\subsection{Acoustic potential energy}

Acoustic potential energy in a cavity, $E_{p}$, is defined as 


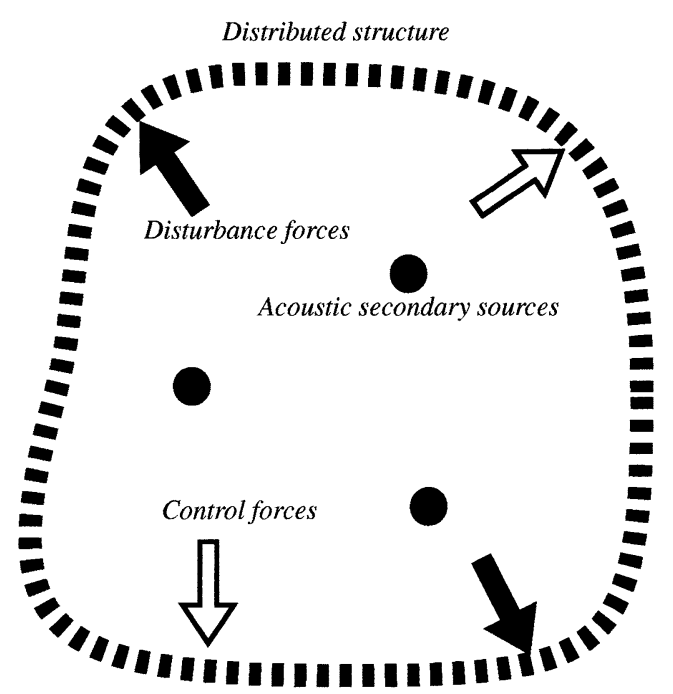

Fig. 1 Acoustic secondary sources in enclosure walled by distributed structure where disturbance and control forces act

$$
E_{p}=\frac{1}{4 \rho c^{2}} \int_{V}\left|p\left(\boldsymbol{r}_{e}\right)\right|^{2} d \boldsymbol{r}_{e}
$$

where $\rho, c$ and $V$ are density of the medium, speed of sound and the area in the cavity respectively. Acoustic pressure at any location $\boldsymbol{r}_{e}$ within the cavity, $p\left(\boldsymbol{r}_{e}\right)$, can be expanded as the sum of modal contributions.

$$
p\left(\boldsymbol{r}_{e}\right)=\sum_{n=1}^{\infty} \psi_{n}\left(\boldsymbol{r}_{e}\right) p_{n} \approx \boldsymbol{\psi}^{\mathrm{T}}\left(\boldsymbol{r}_{e}\right) \boldsymbol{p}
$$

where ${ }^{\mathrm{T}}$ indicates matrix transpose, $\psi_{n}\left(\boldsymbol{r}_{e}\right)$ and $p_{n}$ describe the $n$th modal acoustic shaping function and the modal amplitude respectively. Considering a truncated set of acoustic modes, the vectors $\psi\left(\boldsymbol{r}_{e}\right)$ and $\boldsymbol{p}$ are written as

$$
\begin{aligned}
& \boldsymbol{\psi}\left(\boldsymbol{r}_{e}\right)=\left(\psi_{1}\left(\boldsymbol{r}_{e}\right) \cdots \psi_{N}\left(\boldsymbol{r}_{e}\right)\right)^{\mathrm{T}} \\
& \boldsymbol{p}=\left(p_{1} \cdots p_{N}\right)^{\mathrm{T}}
\end{aligned}
$$

Substituting Eq. (2) into Eq. (1), the acoustic potential energy $E_{p}$ is expressed as

$$
E_{p}=\frac{1}{4 \rho c^{2}} \int_{V} \boldsymbol{p}^{\mathrm{H}} \boldsymbol{\psi}^{*}\left(\boldsymbol{r}_{e}\right) \boldsymbol{\psi}^{\mathrm{T}}\left(\boldsymbol{r}_{e}\right) \boldsymbol{p} d \boldsymbol{r}_{e}
$$

where ${ }^{*}$ and ${ }^{\mathrm{H}}$ represent conjugate and the Hermitian transpose respectively. Introduce that the modal characteristics functions are orthogonal and normalized as

$$
\int_{V} \psi_{m}\left(\boldsymbol{r}_{e}\right) \psi_{n}\left(\boldsymbol{r}_{e}\right) d \boldsymbol{r}_{e}=\left\{\begin{array}{ccc}
0 & \cdots & m \neq n \\
\Lambda_{n} & \cdots & m=n
\end{array}\right.
$$

Thus, Eq. (5) is obtained as

$$
\begin{aligned}
E_{p} & =\frac{1}{4 \rho c^{2}} \sum_{n=1}^{N} \Lambda_{n}\left|p_{n}\right| \\
& =\frac{1}{4 \rho c^{2}} \boldsymbol{p}^{\mathrm{H}} \boldsymbol{\Lambda} \boldsymbol{p}
\end{aligned}
$$

where $\Lambda_{n}$ indicates the $n$th modal mass and matrix $\Lambda$ is defined as

$$
\boldsymbol{\Lambda}=\left(\begin{array}{ccc}
\Lambda_{1} & & \mathbf{0} \\
& \ddots & \\
\mathbf{0} & & \Lambda_{N}
\end{array}\right) \in \boldsymbol{R}^{N \times N}
$$

The acoustic modal amplitude vector $\boldsymbol{p}$ is expanded into the sum of the vector $\boldsymbol{p}_{p}$ contributed by the flexible wall and the vector $\boldsymbol{p}_{s}$ contributed by the acoustic point sources.

$$
\boldsymbol{p}=\boldsymbol{p}_{p}+\boldsymbol{p}_{s}
$$

Substituting Eq. (9) into Eq. (7), the acoustic potential energy $E_{p}$ can be yielded as

$$
\begin{aligned}
E_{p} & =\frac{1}{4 \rho c^{2}}\left(\boldsymbol{p}_{p}+\boldsymbol{p}_{s}^{\mathrm{H}}\right) \boldsymbol{\Lambda}\left(\boldsymbol{p}_{p}+\boldsymbol{p}_{s}\right) \\
& =\frac{1}{4 \rho c^{2}}\left(\boldsymbol{p}_{s}^{\mathrm{H}} \boldsymbol{\Lambda} \boldsymbol{p}_{s}+\boldsymbol{p}_{p}^{\mathrm{H}} \boldsymbol{\Lambda} \boldsymbol{p}_{s}+\boldsymbol{p}_{s}^{\mathrm{H}} \boldsymbol{\Lambda} \boldsymbol{p}_{p}+\boldsymbol{p}_{p}^{\mathrm{H}} \boldsymbol{\Lambda} \boldsymbol{p}_{p}\right)
\end{aligned}
$$

The acoustic pressure $p\left(\boldsymbol{r}_{e}\right)$ excited by a acoustic point source, whose volume velocity is $q\left(\boldsymbol{r}_{o}\right)$, is defined as

$$
p\left(\boldsymbol{r}_{e}\right)=j \omega \rho G\left(\boldsymbol{r}_{e}, \boldsymbol{r}_{0}, \omega\right) q\left(\boldsymbol{r}_{0}\right)
$$

where $G\left(\boldsymbol{r}_{e}, \boldsymbol{r}_{0}, \omega\right)$ represents the Green function in a cavity and is defined as ${ }^{(11)}$

$$
G\left(\boldsymbol{r}_{e}, \boldsymbol{r}_{0}, \omega\right)=c^{2} \sum_{n=1}^{\infty} \frac{\psi_{n}\left(\boldsymbol{r}_{e}\right) \psi_{n}\left(\boldsymbol{r}_{0}\right)}{\Lambda_{n}\left(\omega_{n}^{2}-\omega^{2}+j \eta \omega_{n} \omega\right)}
$$

where $\omega_{n}$ indicates the $n$th acoustic modal frequency, $\omega$ is the excitation frequency, $j$ is the imaginary unit and $\eta$ represents the damping ratio. As the acoustic sources within the cavity are the flexible wall and acoustic point sources, the acoustic pressure $p\left(\boldsymbol{r}_{e}\right)$ can be obtained from Eq. (11).

$$
\begin{gathered}
p\left(\boldsymbol{r}_{e}\right)=j \omega \rho\left\{\int_{S} G\left(\boldsymbol{r}_{e}, \boldsymbol{r}_{p}, \omega\right) v\left(\boldsymbol{r}_{p}\right) d \boldsymbol{r}_{p}\right. \\
\left.+\sum_{i=1}^{N_{s}} G\left(\boldsymbol{r}_{e}, \boldsymbol{r}_{s, i}, \omega\right) q\left(\boldsymbol{r}_{s, i}\right)\right\}
\end{gathered}
$$

where $S$ indicates the area of the flexible wall, $v\left(\boldsymbol{r}_{p}\right)$ represents the velocity at any location $\boldsymbol{r}_{p}$ on the wall and $q\left(\boldsymbol{r}_{s, i}\right)$ describes the volume velocity of any acoustic point source at $\boldsymbol{r}_{s, i}$ within the cavity.

Consequently, each $n$th element of the vector $\boldsymbol{p}_{p}$ and $\boldsymbol{p}_{s}$ are expressed from Eqs. (12) and (13) as

$$
\begin{aligned}
& \boldsymbol{p}_{p}(n)=\frac{j \omega \rho c^{2}}{\Lambda_{n}\left(\omega_{n}^{2}-\omega^{2}+j \eta \omega_{n} \omega\right)} \int_{S} G\left(\boldsymbol{r}_{e}, \boldsymbol{r}_{p}, \omega\right) v\left(\boldsymbol{r}_{p}\right) d \boldsymbol{r}_{p} \\
& \boldsymbol{p}_{s}(n)=\frac{j \omega \rho c^{2}}{\Lambda_{n}\left(\omega_{n}^{2}-\omega^{2}+j \eta \omega_{n} \omega\right)} \sum_{i=1}^{N_{s}} G\left(\boldsymbol{r}_{e}, \boldsymbol{r}_{s, i}, \omega\right) q\left(\boldsymbol{r}_{s, i}\right)
\end{aligned}
$$

The velocity $v\left(\boldsymbol{r}_{p}\right)$ is expanded as the sum of modal contributions: the $n$th vibration modal function $\varphi_{n}\left(\boldsymbol{r}_{p}\right)$ and the modal amplitude $v_{n}$.

$$
v\left(\boldsymbol{r}_{p}\right)=\sum_{n=1}^{\infty} \varphi_{n}\left(\boldsymbol{r}_{p}\right) v_{n} \approx \boldsymbol{\varphi}^{\mathrm{T}}\left(\boldsymbol{r}_{p}\right) \boldsymbol{v}
$$

The vectors $\varphi\left(\boldsymbol{r}_{p}\right)$ and $\boldsymbol{v}$ are defined as

$$
\begin{aligned}
& \boldsymbol{\varphi}\left(\boldsymbol{r}_{p}\right)=\left(\varphi_{1}\left(\boldsymbol{r}_{p}\right) \cdots \varphi_{M}\left(\boldsymbol{r}_{p}\right)\right)^{\mathrm{T}} \\
& \boldsymbol{v}=\left(v_{1} \cdots v_{M}\right)^{\mathrm{T}}
\end{aligned}
$$

The vector $\boldsymbol{v}$ can be written as the sum of a vector $\boldsymbol{v}_{d}$ contributed by disturbance forces and a vector $\boldsymbol{v}_{c}$ contributed by control forces. 


$$
\boldsymbol{v}=\boldsymbol{v}_{d}+\boldsymbol{v}_{c}
$$

The equation of motion on a distributed structure is defined as

$$
L[w(\boldsymbol{r}, t)]+m(\boldsymbol{r}) \ddot{w}(\boldsymbol{r}, t)=\sum_{k=1}^{N_{k}} f\left(\boldsymbol{r}_{k}, t\right) \delta\left(\boldsymbol{r}-\boldsymbol{r}_{k}\right)
$$

In the above equation, $L$ indicates the self differential operator, $w(\boldsymbol{r}, t)$ describes the displacement of the structure at any location $\boldsymbol{r}$ at time $t, m(\boldsymbol{r})$ is the density of mass, $f\left(\boldsymbol{r}_{k}, t\right)$ is the applied force at location $\boldsymbol{r}_{k}$ at time $t, N_{k}$ represents the number of forces' points and $\delta$ describes the Dirac delta function. In the cases where the structure is a plate and the forces drive at frequency $\omega$, Eq. (20) can be rewritten as

$$
D \nabla^{4} w(\boldsymbol{r}) e^{j \omega t}-\omega^{2} \rho_{0} h w(\boldsymbol{r}) e^{j \omega t}=\sum_{k=1}^{N_{k}} f\left(\boldsymbol{r}_{k}\right) \delta\left(\boldsymbol{r}-\boldsymbol{r}_{k}\right) e^{j \omega t}
$$

where $D$ is the flexural rigidity, $\rho_{0}$ is the density of the plate, $h$ is the thickness of the plate and $\nabla^{4}$ is defined as

$$
\nabla^{4}=\nabla^{2} \nabla^{2}=\frac{\partial^{4}}{\partial x^{4}}+2 \frac{\partial^{4}}{\partial x^{2} \partial y^{2}}+\frac{\partial^{4}}{\partial y^{4}}
$$

The displacement $w(\boldsymbol{r})$ is yielded from Eq. (21) as

$$
w(\boldsymbol{r})=\sum_{n=1}^{\infty} \frac{\varphi_{n}(\boldsymbol{r})}{\rho_{o} h\left(\omega_{s, n}^{2}-\omega^{2}\right)} \sum_{k=1}^{N_{k}} \varphi_{n}\left(\boldsymbol{r}_{k}\right) f\left(\boldsymbol{r}_{k}\right)
$$

where $\omega_{s, n}$ is the $n$th vibration modal frequency of the plate. The velocity of the plate $v(\boldsymbol{r}, t)$ is defined as the displacement $w\left(\boldsymbol{r}_{p}\right)$ differentiated with respect to time.

$$
v(\boldsymbol{r}, t)=\frac{\partial}{\partial t} w(\boldsymbol{r}, t)=j \omega \omega(\boldsymbol{r}) e^{j \omega t}=v(\boldsymbol{r}) e^{j \omega t}
$$

Therefore, each $n$th element of the vector $\boldsymbol{v}_{d}$ and $\boldsymbol{v}_{c}$ in Eq. (19) are obtained as

$$
\begin{aligned}
& \boldsymbol{v}_{d}(n)=\frac{j \omega}{\rho_{0} h\left(\omega_{s, n}^{2}-\omega^{2}\right)} \sum_{i=1}^{N_{d}} \varphi_{n}\left(\boldsymbol{r}_{d, i}\right) f\left(\boldsymbol{r}_{d, i}\right) \\
& \boldsymbol{v}_{c}(n)=\frac{j \omega}{\rho_{0} h\left(\omega_{s, n}^{2}-\omega^{2}\right)} \sum_{i=1}^{N_{c}} \varphi_{n}\left(\boldsymbol{r}_{c, i}\right) f\left(\boldsymbol{r}_{c, i}\right)
\end{aligned}
$$

Moreover Eqs. (25) and (26) can be rewritten as

$$
\begin{aligned}
& \boldsymbol{v}_{d}=\boldsymbol{Z}_{d} \boldsymbol{f}_{d} \\
& \boldsymbol{v}_{c}=\boldsymbol{Z}_{c} \boldsymbol{f}_{c}
\end{aligned}
$$

where $\boldsymbol{f}_{d} \in \boldsymbol{C}^{N_{d}}$ is the disturbance forces vector and $\boldsymbol{f}_{c} \in$ $C^{N_{c}}$ is the control forces vector. The matrices $\boldsymbol{Z}_{d} \in C^{N \times N_{d}}$ and $\boldsymbol{Z}_{c} \in \boldsymbol{C}^{N \times N_{c}}$ are the mechanical impedance matrices and their $(n, i)$ th elements are defined as

$$
\begin{aligned}
& \boldsymbol{Z}_{d}(n, i)=\frac{j \omega \varphi_{n}\left(\boldsymbol{r}_{d, i}\right)}{\rho_{0} h\left(\omega_{s, n}^{2}-\omega^{2}\right)} \\
& \boldsymbol{Z}_{c}(n, i)=\frac{j \omega \varphi_{n}\left(\boldsymbol{r}_{c, i}\right)}{\rho_{0} h\left(\omega_{s, n}^{2}-\omega^{2}\right)}
\end{aligned}
$$

Using Eqs. (16), (19), (27) and (28), the acoustic modal amplitude vector $\boldsymbol{p}_{p}$ written as Eq. (14) can be expressed as

$$
\boldsymbol{p}_{p}=\boldsymbol{\Phi} \boldsymbol{Z}_{c} \boldsymbol{f}_{c}+\boldsymbol{\Phi} \boldsymbol{Z}_{d} \boldsymbol{f}_{d}
$$

where the $(n, m)$ th element of the matrix $\boldsymbol{\Phi} \in \boldsymbol{C}^{N \times M}$ is defined as

$$
\boldsymbol{\Phi}(n, m)=\frac{j \omega \rho c^{2}}{\Lambda_{n}\left(\omega_{n}^{2}-\omega^{2}+j \eta \omega_{n} \omega\right)} \int_{S} \psi_{n}\left(\boldsymbol{r}_{p}\right) \varphi_{m}\left(\boldsymbol{r}_{p}\right) d \boldsymbol{r}_{p}
$$

Note the matrix $\boldsymbol{\Phi}$. Each element of the matrix $\boldsymbol{\Phi}$ includes the product of the acoustic modal function $\psi_{n}\left(\boldsymbol{r}_{p}\right)$ of the cavity and the vibration modal function $\varphi_{m}\left(\boldsymbol{r}_{p}\right)$ of the plate so that the cavity has the structural/acoustic coupled system. The couple is, however, weak because the modal functions exist independently in their own fields.

The acoustic modal amplitude vector $\boldsymbol{p}_{s}$ written in Eq. (15) can be rewritten as

$$
\boldsymbol{p}_{s}=\boldsymbol{\Gamma} \boldsymbol{q}_{s}
$$

where the vector $\boldsymbol{q}_{s} \in \boldsymbol{C}^{N_{s}}$ represents the volume velocity vector of the acoustic point sources and the $(n, i)$ th element of the matrix $\boldsymbol{\Gamma} \in \boldsymbol{C}^{N \times N_{s}}$ is defined as

$$
\boldsymbol{\Gamma}(n, i)=\frac{j \omega \rho c^{2}}{\Lambda_{n}\left(\omega_{n}^{2}-\omega^{2}+j \eta \omega_{n} \omega\right)} \psi_{n}\left(\boldsymbol{r}_{s, i}\right)
$$

Therefore, substituting Eqs. (31) and (33) into Eq. (10) yields the acoustic potential energy $E_{p}$ as

$$
\begin{aligned}
E_{p}= & \boldsymbol{q}^{\mathrm{H}} \boldsymbol{A} \boldsymbol{q}+\boldsymbol{f}_{c}^{\mathrm{H}} \boldsymbol{B}^{\mathrm{H}} \boldsymbol{q}+\boldsymbol{f}_{d}^{\mathrm{H}} \boldsymbol{C}^{\mathrm{H}} \boldsymbol{q}+\boldsymbol{q}^{\mathrm{H}} \boldsymbol{B} \boldsymbol{f}_{c}+\boldsymbol{f}_{c}^{\mathrm{H}} \boldsymbol{D} \boldsymbol{f}_{c} \\
& +\boldsymbol{f}_{d}^{\mathrm{H}} \boldsymbol{E}^{\mathrm{H}} \boldsymbol{f}_{c}+\boldsymbol{q}^{\mathrm{H}} \boldsymbol{C} \boldsymbol{f}_{d}+\boldsymbol{f}_{c}^{\mathrm{H}} \boldsymbol{E} \boldsymbol{f}_{d}+\boldsymbol{f}_{d}^{\mathrm{H}} \boldsymbol{F} \boldsymbol{f}_{d}
\end{aligned}
$$

The matrices in Eq. (35) are defined as following expressions.

$$
\left\{\begin{array}{l}
\boldsymbol{A}=\frac{1}{4 \rho c^{2}} \boldsymbol{\Gamma}^{\mathrm{H}} \boldsymbol{\Lambda} \boldsymbol{\Gamma} \in \boldsymbol{R}^{N_{s}} \\
\boldsymbol{B}=\frac{1}{4 \rho c^{2}} \boldsymbol{\Gamma}^{\mathrm{H}} \boldsymbol{\Lambda} \boldsymbol{\Phi} \boldsymbol{Z}_{c} \in \boldsymbol{C}^{N_{s} \times N_{c}} \\
\boldsymbol{C}=\frac{1}{4 \rho c^{2}} \boldsymbol{\Gamma}^{\mathrm{H}} \boldsymbol{\Lambda} \boldsymbol{\Phi} \boldsymbol{Z}_{d} \in \boldsymbol{C}^{N_{s} \times N_{d}} \\
\boldsymbol{D}=\frac{1}{4 \rho c^{2}} \boldsymbol{Z}_{c}^{\mathrm{H}} \boldsymbol{\Phi}^{\mathrm{H}} \boldsymbol{\Lambda} \boldsymbol{\Phi} \boldsymbol{Z}_{c} \in \boldsymbol{R}^{N_{c} \times N_{c}} \\
\boldsymbol{E}=\frac{1}{4 \rho c^{2}} \boldsymbol{Z}_{c}^{\mathrm{H}} \boldsymbol{\Phi}^{\mathrm{H}} \boldsymbol{\Lambda} \boldsymbol{\Phi} \boldsymbol{Z}_{d} \in \boldsymbol{R}^{N_{c} \times N_{d}} \\
\boldsymbol{F}=\frac{1}{4 \rho c^{2}} \boldsymbol{Z}_{d}^{\mathrm{H}} \boldsymbol{\Phi}^{\mathrm{H}} \boldsymbol{\Lambda} \boldsymbol{\Phi} \boldsymbol{Z}_{d} \in \boldsymbol{R}^{N_{d} \times N_{d}}
\end{array}\right.
$$

Note that the matrices $\boldsymbol{A}, \boldsymbol{D}, \boldsymbol{F}$ are real symmetric. Additionally, the acoustic potential energy applied either ANC or AVC is written with the matrices in Eq. (36) as below.

(i ) ANC

$$
E_{p}=\boldsymbol{q}^{\mathrm{H}} \boldsymbol{A} \boldsymbol{q}+\boldsymbol{f}_{d}^{\mathrm{H}} \boldsymbol{C}^{\mathrm{H}} \boldsymbol{q}+\boldsymbol{q}^{\mathrm{H}} \boldsymbol{C} \boldsymbol{f}_{d}+\boldsymbol{f}_{d}^{\mathrm{H}} \boldsymbol{F} \boldsymbol{f}_{d}
$$

(ii) $\mathrm{AVC}$

$$
E_{p}=\boldsymbol{f}_{c}^{\mathrm{H}} \boldsymbol{D} \boldsymbol{f}_{c}+\boldsymbol{f}_{d}^{\mathrm{H}} \boldsymbol{E}^{\mathrm{H}} \boldsymbol{f}_{c}+\boldsymbol{f}_{c}^{\mathrm{H}} \boldsymbol{E} \boldsymbol{f}_{d}+\boldsymbol{f}_{d}^{\mathrm{H}} \boldsymbol{F} \boldsymbol{f}_{d}
$$

Consider the physical meaning of each term in Eq. (35) which expresses the acoustic potential energy. The 1 st term represents the acoustic potential energy when only acoustic point sources are applied. The sum of the 2nd term and the 5th term describes the acoustic potential energy borne by an interaction between the noise radiated from the acoustic point sources and the noise from 
the wall excited by only control forces. The sum of the 3rd and the 8th terms shows the energy borne by an interaction between the noise from the acoustic point sources and the noise from the wall excited by only disturbance forces. The 4th term describes the acoustic potential energy contributed by only control forces. The sum of the 6th and 9th terms describes the energy borne by an interaction between the noise excited by control forces and by disturbance forces. The 7 th term indicates the acoustic potential energy without control.

\subsection{Deriving the optimal control law}

The optimal feed-forward control law to minimize the acoustic potential energy $E_{p}$ can be found by differentiating Eq. (35) with respect to the control vectors, $\boldsymbol{q}_{s}$ and $\boldsymbol{f}_{c}$, and setting the results equal to zero vectors.

Preparatory to doing that, the vectors, $\boldsymbol{q}_{s}$ and $\boldsymbol{f}_{c}$, are expanded into its real and imaginary components as

$$
\begin{aligned}
& \boldsymbol{q}_{s}=\boldsymbol{q}_{s_{-} r}+j \boldsymbol{q}_{s_{-} i} \\
& \boldsymbol{f}_{c}=\boldsymbol{f}_{c_{-} r}+j \boldsymbol{f}_{c_{-} i}
\end{aligned}
$$

Differentiating Eq. (35) with respect to $\boldsymbol{q}_{s_{-} r}, \boldsymbol{q}_{s_{-} i}, \boldsymbol{f}_{c_{-} r}$ and $f_{c \_i}$, the following equations are expressed.

$$
\begin{aligned}
\frac{\partial E_{p}}{\partial \boldsymbol{q}_{s_{-} r}} & =2\left(\boldsymbol{A} \boldsymbol{q}_{s}+\boldsymbol{R e}\left[\boldsymbol{B} \boldsymbol{f}_{c}\right]+\boldsymbol{R} \boldsymbol{e}\left[\boldsymbol{C} \boldsymbol{f}_{d}\right]\right) \\
\frac{\partial E_{p}}{\partial \boldsymbol{q}_{s_{-} i}} & =2\left(\boldsymbol{A} \boldsymbol{q}_{s}+\boldsymbol{R} \boldsymbol{e}\left[\boldsymbol{B} \boldsymbol{f}_{c}\right]+\boldsymbol{R} \boldsymbol{e}\left[\boldsymbol{C} \boldsymbol{f}_{d}\right]\right) \\
\frac{\partial E_{p}}{\partial \boldsymbol{f}_{f_{-} r}} & =2\left(\boldsymbol{D} \boldsymbol{f}_{c}+\boldsymbol{R} \boldsymbol{e}\left[\boldsymbol{B}^{\mathrm{H}} \boldsymbol{q}_{s}\right]+\boldsymbol{R} \boldsymbol{e}\left[\boldsymbol{E} \boldsymbol{f}_{d}\right]\right) \\
\frac{\partial E_{p}}{\partial \boldsymbol{f}_{f_{-} i}} & =2\left(\boldsymbol{D} \boldsymbol{f}_{c}+\boldsymbol{R} \boldsymbol{e}\left[\boldsymbol{B}^{\mathrm{H}} \boldsymbol{q}_{s}\right]+\boldsymbol{R} \boldsymbol{e}\left[\boldsymbol{E} \boldsymbol{f}_{d}\right]\right)
\end{aligned}
$$

where $\boldsymbol{R} \boldsymbol{e}[\mathrm{]}$ indicates the real component of the expression in the bracket. Setting Eqs. (41)-(44) equal to zero vectors, Eqs. (41) and (42) yield Eq. (45), and Eqs. (43) and (44) express Eq. (46)

$$
\begin{aligned}
& \boldsymbol{A} \boldsymbol{q}_{s}+\boldsymbol{B} \boldsymbol{f}_{c}+\boldsymbol{C} \boldsymbol{f}_{d}=\mathbf{0}_{s} \\
& \boldsymbol{D} \boldsymbol{f}_{c}+\boldsymbol{B}^{\mathrm{H}} \boldsymbol{q}_{s}+\boldsymbol{E} \boldsymbol{f}_{d}=\mathbf{0}_{c}
\end{aligned}
$$

where $\mathbf{0}_{s} \in \boldsymbol{R}^{N_{s}}$ and $\mathbf{0}_{c} \in \boldsymbol{R}^{N_{c}}$ describe zero vectors.

Consequently, the optimal feed-forward control law, $\boldsymbol{q}_{s, o p t}$ and $\boldsymbol{f}_{c, o p t}$, to minimize the acoustic potential energy is derived as following expressions.

$$
\begin{aligned}
& \boldsymbol{q}_{s, o p t}=\left(\boldsymbol{A}-\boldsymbol{B} \boldsymbol{D}^{-1} \boldsymbol{B}^{\mathrm{H}}\right)^{-1}\left(\boldsymbol{B} \boldsymbol{D}^{-1} \boldsymbol{E}-\boldsymbol{C}\right) \boldsymbol{f}_{d} \\
& \boldsymbol{f}_{c, \text { opt }}=\left(\boldsymbol{D}-\boldsymbol{B}^{\mathrm{H}} \boldsymbol{A}^{-1} \boldsymbol{B}\right)^{-1}\left(\boldsymbol{B}^{\mathrm{H}} \boldsymbol{A}^{-1} \boldsymbol{C}-\boldsymbol{E}\right) \boldsymbol{f}_{d}
\end{aligned}
$$

Additionally, the optimal control law when either ANC or AVC is applied are also expressed.

$$
\begin{aligned}
& \text { (i) ANC } \\
& \boldsymbol{q}_{s, o_{\text {opt }} s}=-\boldsymbol{A}^{-1} \boldsymbol{C} \boldsymbol{f}_{d}
\end{aligned}
$$

(ii) $\mathrm{AVC}$

$$
\boldsymbol{f}_{c, o p t_{-} c}=-\boldsymbol{D}^{-1} \boldsymbol{E} \boldsymbol{f}_{d}
$$

\section{Zero Control Power}

\subsection{Zero control acoustic power}

Acoustic power radiated from a control acoustic point source $\boldsymbol{r}_{s, i}$ is defined as

$$
P_{w s, i}=\frac{1}{2} \boldsymbol{R} \boldsymbol{e}\left[q_{s, i} p^{*}\left(\boldsymbol{r}_{s, i}\right)\right]
$$

The acoustic pressure $p\left(\boldsymbol{r}_{s, i}\right)$ is expressed from Eqs. (2), (9), (31) and (33) as

$$
\begin{aligned}
p\left(\boldsymbol{r}_{s, i}\right) & =\boldsymbol{\psi}^{\mathrm{T}}\left(\boldsymbol{r}_{s, i}\right) \boldsymbol{p} \\
& =\boldsymbol{\psi}^{\mathrm{T}}\left(\boldsymbol{r}_{s, i}\right)\left\{\boldsymbol{\Phi} \boldsymbol{Z}_{c} \boldsymbol{f}_{c}+\boldsymbol{\Phi} \boldsymbol{Z}_{d} \boldsymbol{f}_{d}+\boldsymbol{\Gamma} \boldsymbol{q}_{s}\right\}
\end{aligned}
$$

Rewrite the optimal control law written as Eqs. (53) and (54).

$$
\begin{aligned}
\boldsymbol{q}_{s, o p t} & =\left(\boldsymbol{A}-\boldsymbol{B} \boldsymbol{D}^{-1} \boldsymbol{B}^{\mathrm{H}}\right)^{-1}\left(\boldsymbol{B} \boldsymbol{D}^{-1} \boldsymbol{E}-\boldsymbol{C}\right) \boldsymbol{f}_{d} \\
& =\boldsymbol{Q} \boldsymbol{f}_{d} \\
\boldsymbol{f}_{c, o p t} & =\left(\boldsymbol{D}-\boldsymbol{B}^{\mathrm{H}} \boldsymbol{A}^{-1} \boldsymbol{B}\right)^{-1}\left(\boldsymbol{B}^{\mathrm{H}} \boldsymbol{A}^{-1} \boldsymbol{C}-\boldsymbol{E}\right) \boldsymbol{f}_{d} \\
& =\boldsymbol{U} \boldsymbol{f}_{d}
\end{aligned}
$$

When the damping in the cavity can be omitted, all elements of the matrix $\boldsymbol{Q} \in \boldsymbol{C}^{N_{s} \times N_{d}}$ are pure imaginary numbers and the matrix $\boldsymbol{U} \in \boldsymbol{C}^{N_{c} \times N_{d}}$ is real. Substituting Eqs. (52)-(54) into Eq. (51), the control acoustic power $P_{w s, i}$ under the optimal control condition is expressed as

$$
\begin{aligned}
P_{w s, i}= & \frac{1}{2} \boldsymbol{R} \boldsymbol{e}\left[q_{s, i} p^{*}\left(\boldsymbol{r}_{s, i}\right)\right] \\
= & \frac{1}{2} \boldsymbol{R} \boldsymbol{e}\left[\boldsymbol { i } _ { s i } ^ { \mathrm { T } } \boldsymbol { Q } \boldsymbol { f } _ { d } \boldsymbol { f } _ { d } ^ { \mathrm { H } } \left\{\boldsymbol{U}^{\mathrm{T}} \boldsymbol{Z}_{d}^{\mathrm{H}} \boldsymbol{\Phi}^{\mathrm{H}}\right.\right. \\
& \left.\left.+\boldsymbol{Z}_{d}^{\mathrm{H}} \boldsymbol{\Phi}^{\mathrm{H}}+\boldsymbol{Q}^{\mathrm{H}} \boldsymbol{\Gamma}^{\mathrm{H}}\right\} \boldsymbol{\psi}\left(\boldsymbol{R}_{s, i}\right)\right]
\end{aligned}
$$

where the vector $\boldsymbol{i}_{s i} \in \boldsymbol{R}^{N_{s}}$ describes a vector whose $i$ th element is 1 and the others are 0.

Note the matrix $\boldsymbol{f}_{d} \boldsymbol{f}_{d}^{\mathrm{H}}$ in Eq. (55). The $i$ th element of the disturbance vector $\boldsymbol{f}_{d}$ can be written with its amplitude $\tilde{f}_{d, i}$ and phase $\phi_{d, i}$.

$$
\boldsymbol{f}_{d}(i)=\tilde{f}_{d, i} e^{-j \phi_{d, i}}
$$

The $(i, j)$ th element of the matrix $\boldsymbol{f}_{d} \boldsymbol{f}_{d}^{\mathrm{H}}$ is written as

$$
\boldsymbol{f}_{d} \boldsymbol{f}_{d}^{\mathrm{H}}(i, j)=\tilde{f}_{d, i} \tilde{f}_{d, j} e^{-j\left(\phi_{d, i}-\phi_{d, j}\right)}
$$

In the cases where the phase between disturbance forces are either in phase or out of phase, the matrix $\boldsymbol{f}_{d} \boldsymbol{f}_{d}^{\mathrm{H}}$ is a real matrix. Thus the value in [ ] in Eq. (55) is a pure imaginary number because all the terms in it excluding the matrix $\boldsymbol{Q}$ are real matrices. Therefore, Eq. (55) which calculates the control acoustic power becomes zero.

The above result shows that the control acoustic power radiated from each acoustic point source becomes zero under the optimal control condition subject to the phase between disturbance forces to be either in phase or out of phase. However, the zero control power phenomenon appears when the control law is written with the pure imaginary matrix $\boldsymbol{Q}$ and the real matrix $\boldsymbol{P}$ such as Eqs. (53) and (54), namely, the phenomenon is a necessary condition to minimize the acoustic potential energy in the cavity. 


\subsection{Zero control vibration power}

The vibration power infused by control actuator located at $\boldsymbol{r}_{c, i}$ into the structure is defined as

$$
P_{s c, i}=\frac{1}{2} \boldsymbol{R e}\left[f_{c, i} v^{*}\left(\boldsymbol{r}_{c, i}\right)\right]
$$

where $f_{c, i}$ indicates the force of the actuator. Substituting Eqs. (19), (27) and (28) into Eq. (16) yields the velocity $v\left(\boldsymbol{r}_{c, i}\right)$ as the following expression.

$$
\begin{aligned}
v\left(\boldsymbol{r}_{c, i}\right) & =\boldsymbol{\varphi}^{\mathrm{T}}\left(\boldsymbol{r}_{c, i}\right) \boldsymbol{v} \\
& =\boldsymbol{\varphi}^{\mathrm{T}}\left(\boldsymbol{r}_{c, i}\right)\left\{\boldsymbol{Z}_{d} \boldsymbol{f}_{d}+\boldsymbol{Z}_{c} \boldsymbol{f}_{c}\right\}
\end{aligned}
$$

Substituting Eqs. (54) and (59) into Eq. (58), the control vibration power $P_{w c, i}$ under the optimal control condition is expressed as

$$
\begin{aligned}
P_{s c, i} & =\frac{1}{2} \boldsymbol{R} \boldsymbol{e}\left[f_{c, i} v^{*}\left(\boldsymbol{r}_{c, i}\right)\right] \\
& =\frac{1}{2} \boldsymbol{R} \boldsymbol{e}\left[\boldsymbol{i}_{c i}^{\mathrm{T}} \boldsymbol{U} \boldsymbol{f}_{d} \boldsymbol{f}_{d}^{\mathrm{H}}\left\{\boldsymbol{Z}_{d}^{\mathrm{H}}+\boldsymbol{U}^{\mathrm{T}} \boldsymbol{Z}_{d}^{\mathrm{H}}\right\} \boldsymbol{\varphi}\left(\boldsymbol{r}_{c, i}\right)\right]
\end{aligned}
$$

where the vector $\boldsymbol{i}_{c i} \in \boldsymbol{R}^{N_{c}}$ is a vector whose $i$ th element is 1 and the others are 0 . Assume the matrix $\boldsymbol{f}_{d} \boldsymbol{f}_{d}^{\mathrm{H}}$ to be a real matrix as well as in the section 3.1. Thus, the value in the bracket in Eq. (60) is a pure imaginary number because the matrix $\left\{\boldsymbol{Z}_{d}^{\mathrm{H}}+\boldsymbol{U}^{\mathrm{T}} \boldsymbol{Z}_{c}^{\mathrm{H}}\right\}$ is pure imaginary and the other terms are real, namely, the Eq. (60) becomes zero.

Therefore, it is demonstrated that the control vibration power of each control force becomes zero under the optimal control condition when the phase between disturbance forces is in phase or out of phase. The phenomenon, however, appears when the optimal control law is written with a real matrix $\boldsymbol{U}$ as in Eq. (54). In other words, the phenomenon is a necessary condition for the optimal control condition where the acoustic potential energy is minimized.

\section{Numerical Analysis}

In this chapter, disturbance attenuation of the acoustic potential energy achieved by both active noise control and active vibration control are demonstrated from a viewpoint of a numerical analysis with a cavity illustrated in Fig. 2. The cavity is a cuboid bone and its top wall is flexible and the others are rigid. The flexible wall is a $12 \mathrm{~mm}$ thick simply supported steel panel and excited by a disturbance force located at $(0.1 \mathrm{~m}, 0.1 \mathrm{~m}, 1.2 \mathrm{~m})$ and a control force located at $(0.7 \mathrm{~m}, 1.7 \mathrm{~m}, 1.2 \mathrm{~m})$. A secondary acoustic point source is set at $(0.8 \mathrm{~m}, 1.7 \mathrm{~m}, 1.1 \mathrm{~m})$ within the cavity. The control target frequency band is between 30 and $200 \mathrm{~Hz}$. Table 1 lists the resonance frequencies of the structural modes of the flexible wall and acoustic modes of the cavity.

Figure 3 displays the frequency characteristics of acoustic potential energy before and after control. Figure 3 (a) depicts applying only ANC, Fig. 3 (b) shows applying only AVC, Fig. 3 (c) illustrates applying both ANC and AVC. The thin line and the thick line represent the

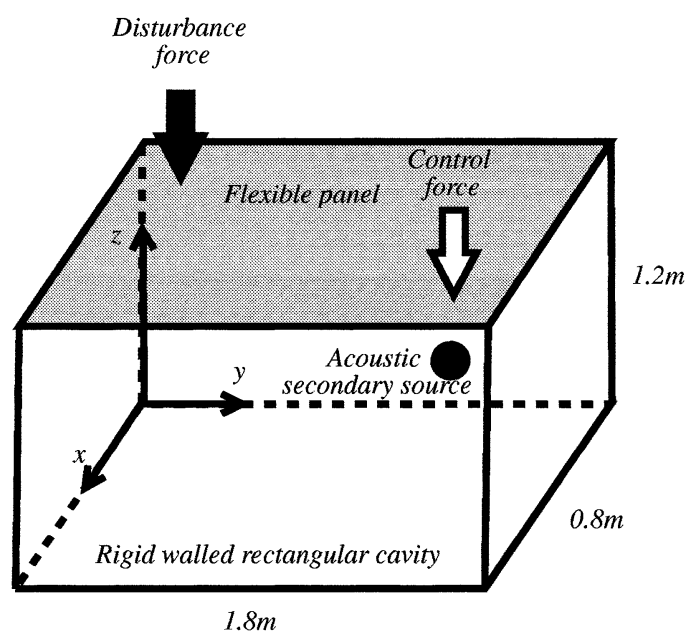

Fig. 2 Enclosure model for a numerical analysis

Table 1 Mode number and modal frequency of the enclosure and panel used for numerical analysis

\begin{tabular}{|c|c|c|c|c|c|c|c|}
\hline No. & Mode & Mode & Frequency & No. & Mode & Mode & Frequency \\
\hline 0 & & $(0,0,0)$ & 0.00 & 5 & & $(0,0,1)$ & 138.10 \\
\hline 1 & $(1,1)$ & & 54.99 & 6 & & $(0,1,1)$ & 165.98 \\
\hline 2 & $(1,2)$ & & 82.20 & 7 & & $(0,2,0)$ & 184.14 \\
\hline 3 & & $(0,1,0)$ & 92.07 & 8 & $(1,4)$ & & 191.05 \\
\hline 4 & $(1,3)$ & & 127.56 & 9 & $(2,1)$ & & 192.75 \\
\hline
\end{tabular}

acoustic potential energy before and after control respectively. Applying only ANC (see Fig. 3 (a)), the acoustic potential energy near acoustic modal resonance frequencies are decreased. Applying only AVC (see Fig. 3(b)), reducing the acoustic potential energy can be achieved through the target frequency band. Using both ANC and AVC (see Fig. 3(c)), the disturbance attenuation can be achieved at whole control target frequencies.

Next, consider the control acoustic power and control vibration power. Figure 4 represents changes of the control powers when the phase of the controller is changed subject to fixed gain; Fig. 4 (a) shows the change of control acoustic power, Fig. 4(b) indicates the change of control vibration power. Under the optimal control condition (the phase is 0 degree), both control acoustic power and control vibration power are zero. The control acoustic power changes from positive to negative, and the control vibration power changes from negative to positive at optimal control condition. These phenomena are illustrated with intensity distributions in Figs. 5 and 6. Figure 5 shows the acoustic intensity distributions on an $x-y$ plane including the control acoustic source, and Fig. 6 represents the vibration intensity distributions on the plate. In these two figures, (a) shows intensity distributions without any control, (b) depicts intensity distributions when the phase is 

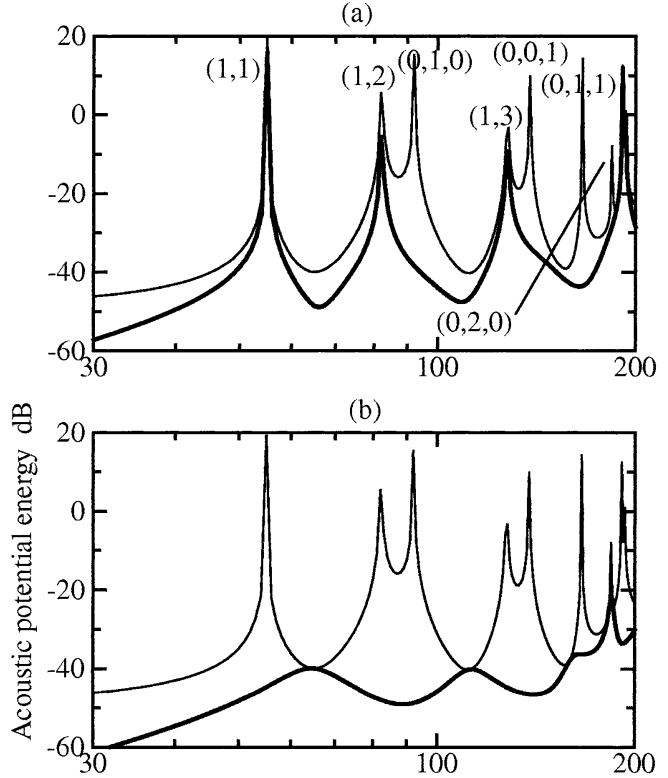

(c)

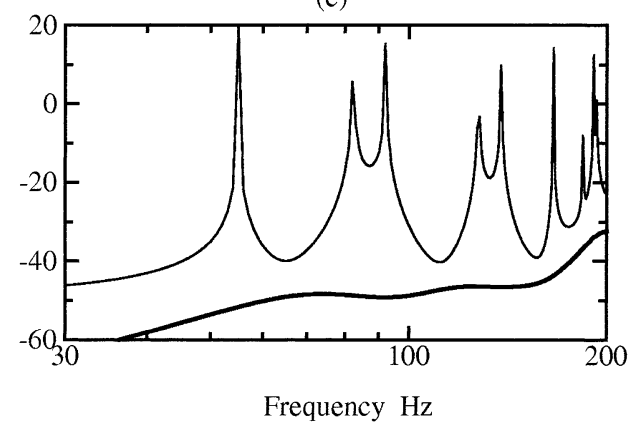

Fig. 3 Frequency characteristics of acoustic potential energy before and after control; -: without control, - : with control, (a) with ANC, (b) with AVC, (c) with both ANC and AVC

delayed for 10 degrees from optimal value, (c) shows intensity distributions at the optimal control condition, (d) illustrates intensity distributions when the phase is led by 10 degrees from optimal value, $\bullet$ indicates the control acoustic source, $\nabla$ represents the location of the disturbance force and $\circ$ describes the location of the control actuator. Before control (see Figs. 5 (a) and 6(a)), the acoustic energy and vibration energy do not flow due to the plate and the cavity dominated by standing waves. When the phase is not optimum (see (b) and (d) of Figs. 5 and 6), the intensities near controllers behave as if the controllers are sources or sinks of the energy. These behaviours describe that the control powers are positive or negative. On the other hand, under the optimal control condition (see Figs. 5 (c) and 6(c)), the acoustic intensities and vibration intensities do not exist as well as without control, namely, both control acoustic power radiated from the control acoustic source and control vibration power infused by the control actuator are zero.

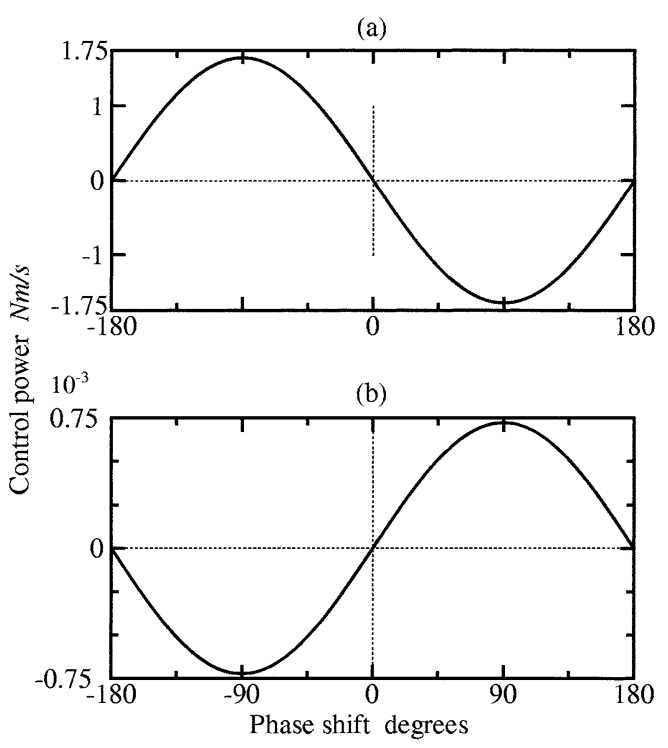

Fig. 4 Control power in the vicinity of optimal control; (a) control acoustic power, (b) control vibration power (a)

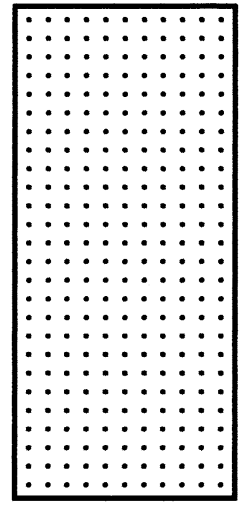

(c)

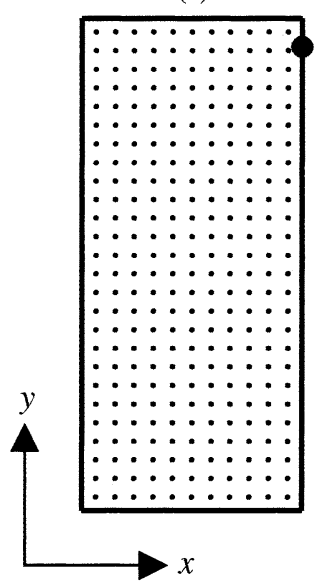

(b)

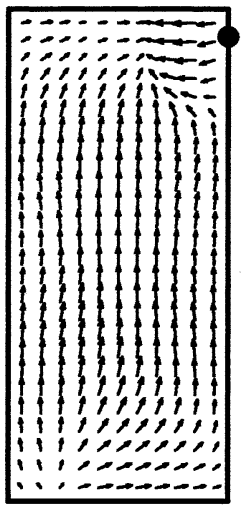

(d)

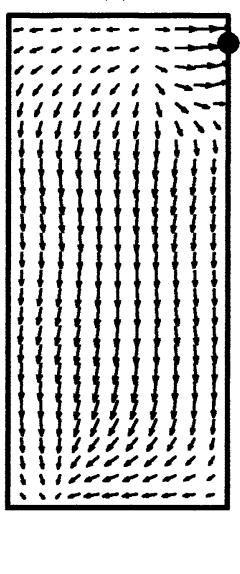

Fig. 5 Acoustic intensity distribution on the $x-y$ plane at the height of the acoustic secondary source location; •: acoustic secondary source, (a) without control, (b) in the vicinity of optimal condition where phase is lagged by 10 degrees from optimality, (c) with control at the optimal condition, (d) in the vicinity of optimal condition where phase is led by degrees from optimality 
(a)

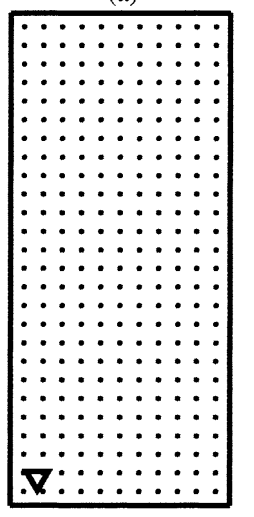

(c)

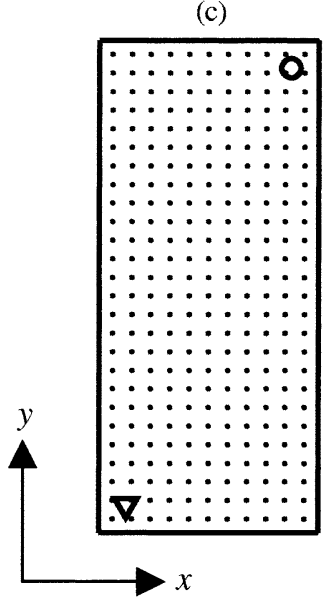

(b)

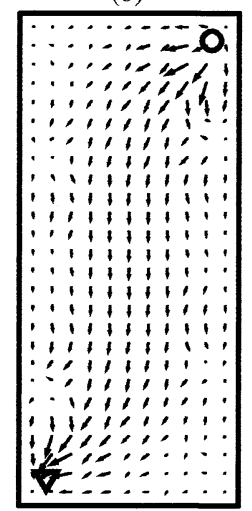

(d)

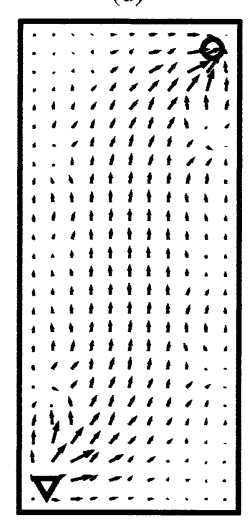

Fig. 6 Vibration intensity distribution on the panel; $\nabla$ : disturbance point, o: control point, (a) without control, (b) in the vicinity of optimal condition where phases is lagged by 10 degrees from optimality, (c) with control at the optimal condition, (d) in the vicinity of optimal condition where phase is led by 10 degrees from optimality

\section{Conclusions}

This paper presented the minimization of the acoustic potential energy in a cavity by using both active noise control and active vibration control. The summarization of this investigation is as follow:

1. The optimal control law for minimizing the acoustic potential energy in a cavity which consists of excited walls by both active noise control and active vibration control has been derived.

2. It has been shown that the control acoustic power radiated from each control acoustic source always becomes zero when the acoustic potential energy is minimized. Likewise, the control vibration power infused by each control actuator. In addition, these zero control power phenomena are found to be the necessary condition

for the optimal condition.

3. From a viewpoint of a numerical analysis, the disturbance attenuation which is achieved through active noise control and active vibration control has been illustrated.

4. The physical meaning of the zero control power phenomena has been demonstrated by acoustic intensity distributions and vibration intensity distributions.

\section{References}

( 1 ) Nelson, P.A., Curtis, A.R.D., Elliott, S.J. and Bullmore, A.J., The Minimum Power Output of Free Field Point Sources and the Active Control of Sound, J. Sound Vib., Vol.116, No.3 (1987), pp.397-414.

(2) Tanaka, N. and Kobayashi, K., Active Noise Control of Structure-Borne Noise Radiated from a Planar Structure, Trans. Jpn. Soc. Mech. Eng., (in Japanese), Vol.67, No.660, C (2001), pp.2528-2535.

( 3 ) Fuller, C.R., Active Control of Sound Transmission/Radiation from Elastic Plates by Vibration Inputs. I:Analysis, J. Sound Vib., Vol.136, No.1 (1990), pp.115.

(4) Kobayashi, K. and Tanaka, N., Minimization of the Acoustic Power Radiated from a Vibrating Panel Using Both Active Noise Control and Active Vibration Control, Trans. Jpn. Soc. Mech. Eng., (in Japanese), Vol.68, No.669, C (2002), pp.1420-1427.

( 5 ) Nelson, P.A., Curtis, A.R.D., Elliott, S.J. and Bullmore, A.J., The Active Minimization of Harmonic Enclosed Sound Fields, Part1: Theory, J.Sound Vib., Vol.117, No.1 (1987), pp.1-13.

( 6 ) Nelson, P.A., Curtis, A.R.D., Elliott, S.J. and Bullmore, A.J., The Active Minimization of Harmonic Enclosed Sound Fields, Part2: A Computer Simulation, J.Sound Vib., Vol.117, No.1 (1987), pp.15-33.

( 7 ) Nelson, P.A., Curtis, A.R.D., Elliott, S.J. and Bullmore, A.J., The Active Minimization of Harmonic Enclosed Sound Fields, Part3: Experimental Verification, J.Sound Vib., Vol.117, No.1 (1987), pp.35-58.

( 8 ) Kim, S.M. and Brennan, M.J., Active Control of Harmonic Sound Transmission into an Acoustic Enclosure Using Both Structural and Acoustic Actuators, J.Acoust.Soc.Am., Vol.107, No.5 (2000), pp.25232534.

( 9 ) Snyder, S.D. and Hansen, D.H., The Design of Systems to Control Actively Periodic Sound Transmission into Enclosed Spaces, Part1: Analytical Models, J.Sound Vib., Vol.170, No.4 (1994), pp.433-449.

(10) Snyder, S.D. and Hansen, C.H., The Design of Systems to Control Actively Periodic Sound Transmission into Enclosed Spaces, Part2: Mechanisms and Trends, J.Sound Vib., Vol.170, No.4 (1994), pp.451-472.

(11) Hansen, C.H. and Snyder, S.D., Active Control of Noise and Vibration, E\&FN SPON, (1997). 